\title{
Erratum to: Plant Bioinformatics: Decoding the Phyta
}

\author{
Khalid Rehman Hakeem, Adeel Malik, Fazilet Vardar-Sukan, \\ and Munir Ozturk
}

\section{Erratum to:}

K.R. Hakeem et al. (eds.), Plant Bioinformatics, https://doi.org/10.1007/978-3-319-67156-7

This book was inadvertently published with incorrect affiliation of Co-editor Adeel Malik.

This has now been amended in the copyright page and Chapter 11 as below:

Adeel Malik

Department of Microbiology and Molecular Biology

Chungnam National University

Daejeon, South Korea 